\title{
General Search for New Physics at HERA
}

\author{
Gerhard Brandt ${ }^{* \dagger}$ \\ Deutsches Elektronen-Synchrotron DESY, D-22603 Hamburg \\ E-mail: gbrandtemail.desy.de
}

\begin{abstract}
A model-independent search for deviations from the Standard Model prediction using the full $e^{ \pm} p$ data sample collected by the $\mathrm{H} 1$ experiment at HERA is presented. All event topologies involving isolated electrons, photons, muons, neutrinos and jets with transverse momenta $P_{T}$ above $20 \mathrm{GeV}$ are investigated in a single analysis. Events are assigned to exclusive classes according to their final state. A dedicated algorithm is used to search for deviations from the Standard Model in the distributions of the scalar sum of transverse momenta or the invariant mass of final state particles and to quantify their significance. Variables related to angular distributions and energy sharing between final state particles are also introduced to study the final state topologies. No significant deviation from the Standard Model expectation is observed in the phase space covered by this analysis.
\end{abstract}

European Physical Society Europhysics Conference on High Energy Physics, EPS-HEP 2009, July 16 - 222009

Krakow, Poland

\footnotetext{
* Speaker.

${ }^{\dagger}$ For the $\mathrm{H} 1$ and ZEUS collaborations.
} 


\section{Analysis Method}

At HERA electron-proton collisions ${ }^{1}$ at centre-of-mass energies of up to $319 \mathrm{GeV}$ provide a testing ground for the Standard Model (SM) complementary to $e^{+} e^{-}$and $p \bar{p}$ scattering. A purely signature based search for new physics beyond the Standard Model in the HERA data, corresponding to an integrated luminosity of $463 \mathrm{pb}^{-1}$, is presented [1].

The analysis investigates all possible high- $P_{T}$ topologies with at least two particles: electrons (e), photons $(\gamma)$, muons $(\mu)$ and jets (j), identified in a common phase space $10^{\circ}<\theta<140^{\circ}$ and $P_{T}>20 \mathrm{GeV}$. A neutrino $(v)$ is identified if the event has missing transverse momentum above $20 \mathrm{GeV}$ and $\left(E-P_{z}\right)<48 \mathrm{GeV}$. All particles are required to be isolated with respect to each other by a minimum distance $R>1$ in $(\eta, \phi)$ space, where $\eta$ is the pseudorapidity and $\phi$ is the azimuthal angle. The events are sorted depending on the number and type of particles into exclusive event classes. All possible event classes with at least two bodies are investigated. The event yields for all event classes are presented for the data and SM expectation in figure 1 for $e^{+} p$ and $e^{-} p$ collisions. All event classes with observed data events or with a SM expectation greater than 0.01 events are shown. Events are observed in 27 classes and a good description of the number of observed data events by the SM prediction is seen in each class.

The distributions of the scalar sum of transverse momenta $\sum P_{T}$ and of the invariant mass $M_{\text {all }}$ of all bodies are investigated. The data are in agreement with the SM prediction. In particular, multiple jets topologies, which are sensitive to QCD radiation, are well described by the simulation. The final state topologies are also evaluated in terms of angular distributions $\cos \theta_{\text {lead }}^{*}$ and energy ratios $X_{\text {lead }}$, which are sensitive to spin and decay properties of hypothetical high mass particles, as demonstrated in figure 2. The event topology is well described by the SM.

\section{Statistical Interpretation}

In order to quantify the level of agreement between the data and the SM expectation and to identify regions of possible deviations, a dedicated search algorithm developed for a general search in HERA I data at the $\mathrm{H} 1$ experiment is employed [2]. All possible regions in the $\sum P_{T}, M_{\text {all }}$, $\cos \theta_{\text {lead }}^{*}$ and $X_{\text {lead }}$ distributions are considered. The number of data events, the SM expectation and its total systematic uncertainty are calculated for each region. A statistical estimator $p$ based on the Poissonian pdf to account for statistical fluctuation, convoluted with a Gaussian pdf to account for the total systematical uncertainty is defined. The value $p$ gives an estimate of the probability for the data to fluctuate up or down from the background prediction in the region considered. The region of most interest, defined as the region of greatest deviation, is the region having the smallest $p$-value, $p_{\min }$. A probability $\hat{P}$ that a fluctuation with a $p$-value at least as small as $p_{\min }$ occurs anywhere in the distribution is estimated using Monte Carlo experiments based on the SM expectation and recalculating $p_{\min }^{\mathrm{SM}}$ on them. The fraction of experiments with a $p_{\min }^{\mathrm{SM}}$ smaller than $p_{\min }$ gives $\hat{P}$. Finally, the overall degree of agreement with the SM is further quantified by doing many Monte Carlo experiments simulating a full set of hypothetical data histograms and rerunning the above algorithms. The distribution of probabilities of observing an event class with a given $\hat{P}$ value is

\footnotetext{
${ }^{1}$ In the following the term "electron" is used generically to refer to both electrons and positrons, unless otherwise stated.
} 
r

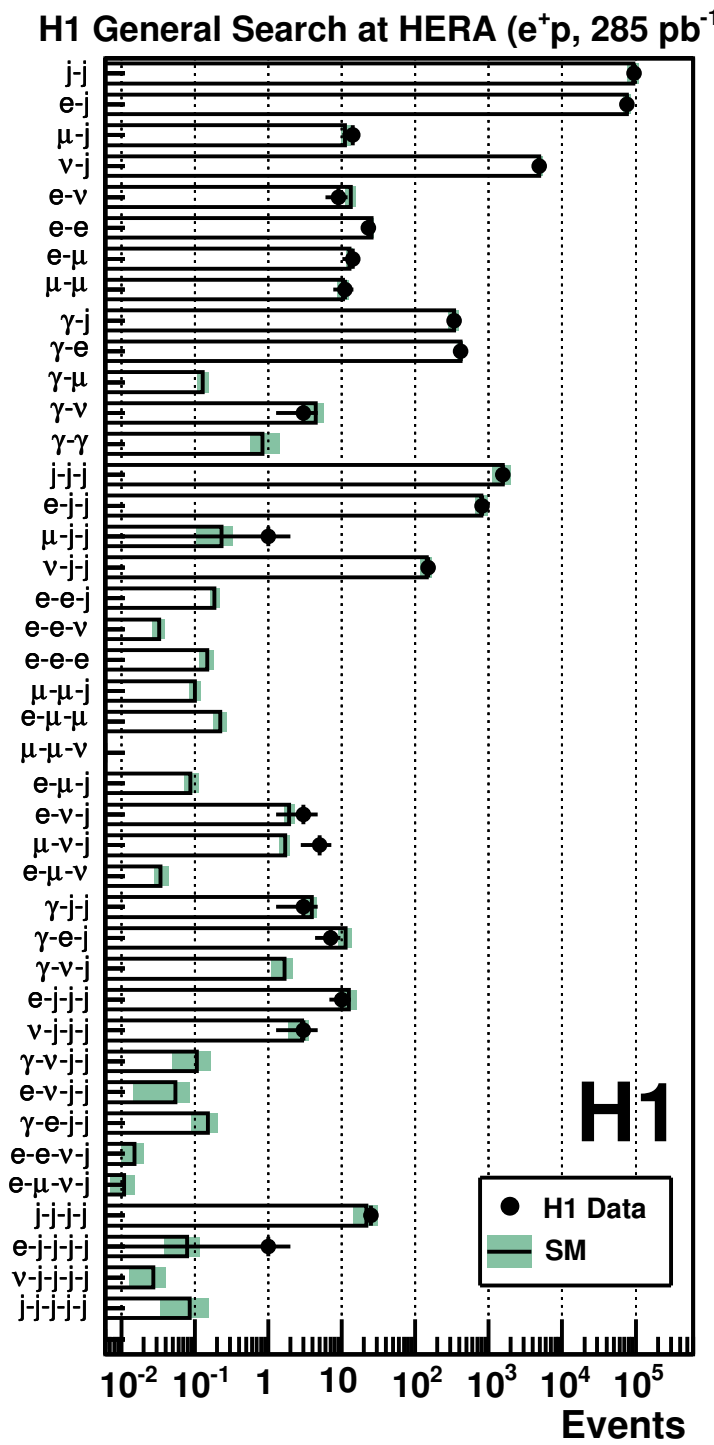

H1 General Search at HERA (e-p, $\left.178 \mathrm{pb}^{-1}\right)$

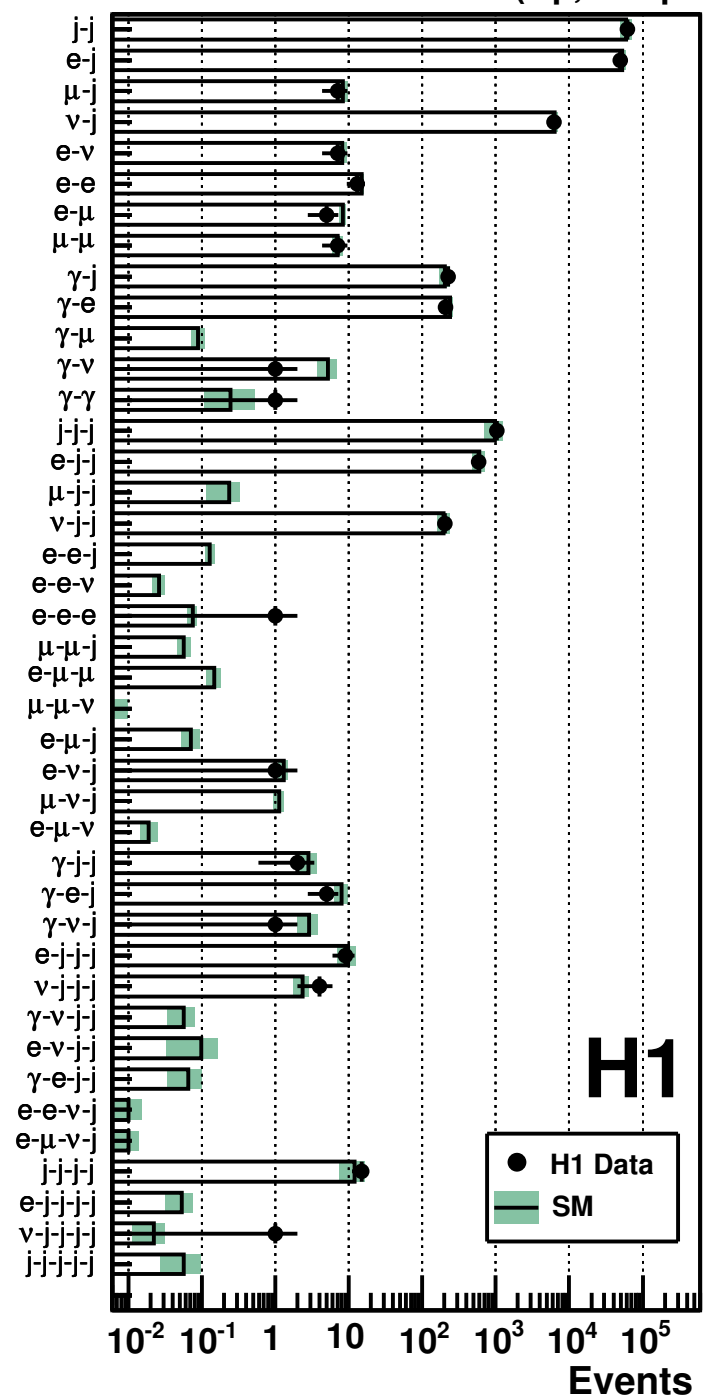

Figure 1: The data and the SM expectation for all event classes with observed data events or a SM expectation greater than 0.01 events for $e^{+} p$ collisions (left) and $e^{-} p$ collisions (right). The error bands on the predictions include model uncertainties and experimental systematic errors added in quadrature.

formed from the $\hat{P}^{S M}$ values obtained from all MC experiments. This distribution is compared to the $\hat{P}$ values observed in the data and gives the global significance of the observed deviations. The most significant deviation from SM predictions is measured in $e^{+} p$ collisions in the $e-e$ event class with $\hat{P}=0.0035$. In the corresponding region $\left(110<M_{\text {all }}<120 \mathrm{GeV}\right)$ five data events are found while $0.43 \pm 0.04$ are expected. The global probability to find in the $e^{+} p$ data at least one class with a $\hat{P}$ value smaller than observed in the $e$-e event class is $12 \%$. These events were also observerd in an analysis of multi-lepton topologies [6]. 
H1 General Search at HERA $\left(e^{ \pm} p, 463 \mathrm{pb}^{-1}\right)$

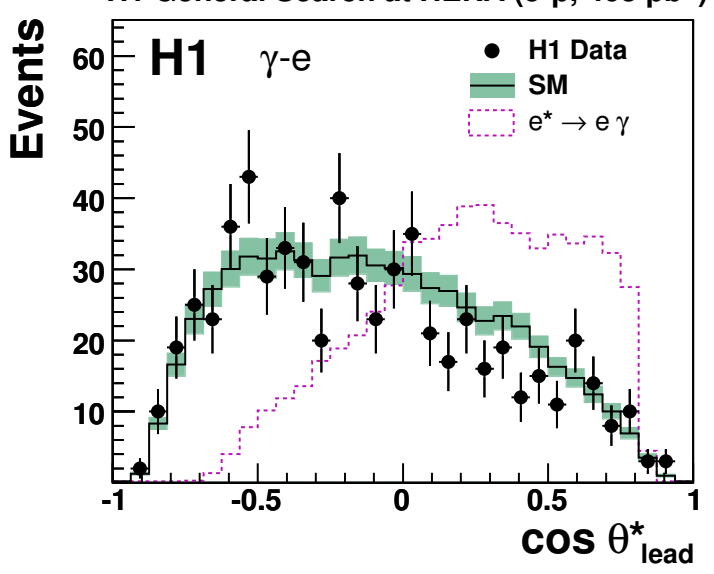

\section{H1 General Search at HERA $\left(e^{ \pm} p, 463 \mathrm{pb}^{-1}\right)$}

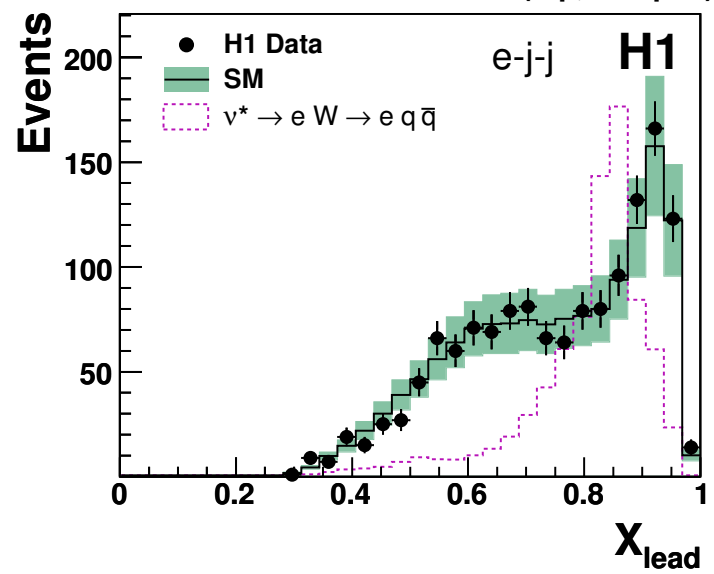

Figure 2: The $\cos \theta_{\text {lead }}^{*}$ distribution in the $\gamma$-e event class (a) and the $X_{\text {lead }}$ distribution in the $e j j$ event class (b). The points correspond to the observed data events and the histograms to the SM expectation. The error bands on the SM prediction include model uncertainties and experimental systematic errors added in quadrature. The dashed line represents, with an arbitrary normalisation, the distribution corresponding to an exotic resonance with a mass of $200 \mathrm{GeV}\left(e^{*}[4]\right.$ (left) and $v^{*}[5]$ (right)).

\section{Summary}

A model-independent search for deviations from the Standard Model prediction using the full $e^{ \pm} p$ data sample collected by the $\mathrm{H} 1$ experiment at HERA has been performed. No significant deviation from the SM expectation has been found, demonstrating the good understanding of high$P_{T}$ processes at HERA. This search constitutes the most comprehensive general search for new physics at high-energy colliders.

\section{References}

[1] F. D. Aaron et al. [H1 Collaboration], "A General Search for New Phenomena at HERA”, Phys. Lett. B 674 (2009) 257 [arXiv:0901.0507].

[2] A. Aktas et al. [H1 Collaboration], "A general search for new phenomena in ep scattering at HERA", Phys. Lett. B 602 (2004) 14 [hep-ex/0408044].

[3] A. Aktas et al. [H1 Collaboration], Phys. Lett. B 629 (2005) 9 [hep-ex/0506044].

[4] F. D. Aaron et al. [H1 Collaboration], Phys. Lett. B 666 (2008) 131 [arXiv:0805.4530].

[5] F. D. Aaron et al. [H1 Collaboration], Phys. Lett. B 663 (2008) 382 [arXiv:0802.1858].

[6] F. D. Aaron et al. [H1 Collaboration], Phys. Lett. B 668 (2008) 268 [arXiv:0806.3987]. 